\title{
Advances in diagnosis of schistosomiasis.
}

\author{
Weifeng $G^{1}$, Joseph $W M^{2}$, Zhisheng $D^{3,4,5,6^{*}}$, Yumin $Z^{1^{*}}$, Wei $H^{3,4,5,6}$ \\ ${ }^{1}$ Department of Parasitology, School of Basic Medicine, Guilin Medical University, Guilin, Guangxi, P.R. China \\ ${ }^{2}$ Veterinary Epidemiologist AU-IBAR/ICPALD, African Union-Interafrican Bureau for Animal Resources, Nairobi, \\ Kenya \\ ${ }^{3}$ Key Laboratory on Biology of Parasite and Vector, Ministry of Health, Shanghai, China \\ ${ }^{4}$ National Center for International Research on Tropical Diseases, Shanghai, China \\ ${ }^{5}$ WHO Collaborating Center for Tropical Diseases, Shanghai, China \\ ${ }^{6}$ National Institute of Parasitic Diseases, Chinese Center for Disease Control and Prevention, Shanghai, P.R. China
}

\begin{abstract}
Schistosomiasis is a kind of globally infectious tropical disease that endangers human health and hinders social development in modern society. The main epidemic species is Schistosoma japonicum in China. Occasionally cases of imported schistosomiasis are reported. This article provides new insights to the research of clinical diagnosis and treatment by summarizing the common diagnosis methods of schistosomiasis in China, such as routine examination, pathogen detection, immunological tests, nucleic acid tests and medical imaging examination.
\end{abstract}

Keywords: Schistosomiasis, Diagnosis, Advances.

Accepted on March 8, 2018

\section{Introduction}

Schistosomiasis is a worldwide infectious parasitic disease that is prevalent in the tropics and subtropics and seriously endangers human health. According to the map of the global distribution of schistosomiasis published by WHO in 2014, nearly 200 million people are infected with schistosomiasis worldwide. The disease is prevalent in 74 countries and regions worldwide, especially in Asia, Africa and Latin America [1,2]. There are 5 kinds of mainly pathogenic Schistosoma in human society, include Schistosoma japonicumSchistosoma mansoniSchistosoma haematobiumSchistosoma intercalatum and Schistosoma mekongi. Schistosomiasis caused by Schistosoma japonicumSchistosoma mansoni and Schistosoma haematobium is the most widespread and most harmful [3]. Most common in China is mainly Schistosoma japonicum that is prevalent in the Yangtze River valley and parts of the South China.

Adult Schistosoma japonicum exist mainly in the human intestinal mesenteric vein and portal vein system. Common gastrointestinal symptoms among patients include diarrhea,abdominal pain and bloody purulent stool and tenesmus in extreme cases. As the disease progresses, severe symptoms such as hepatomegaly, debilitation and maransis occur. Egg sedimentation in mucosa and submucosa of segmented intestine and rectum leads intestinal wall microvascular obstruction, ultimately leading to intestinal erosion and elkosis, including polyps or intestinal stenosis [4].

Eggs deposited in portal area and soluble antigens from eggs could stimulate macrophage to release a series of cytokine, promoting production of a large number of collagen fibres 14 deposited in the liver [5], hence causing liver fibrosis. The process gradually increases over time [6]. Schistosoma haematobium is the main parasite in human urinary system, mainly manifesting scar formation. Patients of Schistosoma haematobium always manifest urological symptoms such as haemuresisdysuria, nephrohydrops and even renal failure $[7,8]$. Whereas the aforementioned are the main symptoms, eggs deposited in the bladder are also easily misdiagnosed as bladder tumors [9]. There is need to pay more attention to the serious consequences caused by the heterotopia of schistosomiasis. Early diagnosis of schistosomiasis is particularly important.

\section{Routine examination}

The relative specificity changes associated with blood routine examination in cases of schistosomiasis is eosinophilia. In addition, Schistosomiasis cases have non-specific increase in white blood cells with counts usually ranging up to 10-30 x $10^{9} / \mathrm{L}$ and with eosinophil ratio between 0.15 and 0.20 [10]. However, some clinical reports indicate that schistosomiasis patients show no changes in eosinophils count [11]. Therefore, eosinophil counts can only assist in the diagnosis of acute schistosomiasis. Schistosomiasis should not be ruled out when eosinophil counts increase, instead define diagnosis should depend on pathogen detection.

\section{Pathogen detection}

Pathogen detection relies on checking the patient's excrement or the use of colonoscopy and other equipment in inspecting common parasitic sites of schistosome to look for parasites or 
eggs. This is the gold standard for the diagnosis of schistosomiasis. Excrement examination microscopic examination of urine sediment and colonoscopy Biopsy are the common methods for pathogen detection.

Excrement examination: Excrement examination is intended to detect Schistosoma eggs or incubation miracidia from patients' excrement, and that constitute the basis for diagnosis. It includes direct smear method, kato-katz, natural precipitation method, nylon sets eggs method, Parasep feces centrifuge tube method and cercariae incubation method. Among them, the direct smear method is relatively simple but of low sensitivity, only sensitive to patients with acute schistosomiasis. Kato-katz is of slightly higher sensitivity than the direct smear method, and is the usual method employed in general surveys in affected areas. Natural sedimentation method relies on the characteristics of schistosoma eggs. A large proportion of eggs easily precipitate to enhance the detection rate of schistosoma eggs under microscopy. In both Nylon sets eggs method and Parasep feces centrifuge tube method [12] , filtering, emulsifying and centrifuging faecal samples is conducted in order to get fecal sediment for microscopic examination. This is intended to increase the detection rate of Schistosoma eggs during fecal examination. The principle of cercariae incubation method is detection of miracidia inside mature Schistosoma japonica eggs. Incubation under suitable temperature and light enables detection of miracidia in the sample, hence confirming the diagnosis of schistosomiasis. This is usually the diagnostic method employed during general surveys in affected areas.

Microscopic examination of urine sediment: Schistosoma haematobium mainly infects the human urinary system. Schistosoma haematobium microfilarias are usually detected in the urine when eggs exist in the bladder [13]. Therefore, urine samples should be collected for suspected schistosomiasis patients and examined through the microscope to establish presence of schistosoma haematobium eggs in the urine sediment. This is the basis for the diagnosis of imported schistosomiasis [14].

Colonoscopy biopsy: For suspected schistosomiasis patients manifesting clear gastrointestinal symptoms, a biopsy of the intestinal mucosal tissue of the lesion can be obtained by colonoscopy to diagnose presence of schistosome eggs [15].

\section{Immunological tests}

Immunological testing is relatively simple with high sensitivity. The principle is to monitor the antigen and antibody release from schistosome. The common immunological tests include intradermal test (ID), indirect hemagglutination assay (IHA), enzyme-linked immunosorbent assay (ELISA), dipstick dye immuno-assay (DDIA), circunoval precipitin test (COPT), dot immunogold filtration assay (DIGFA), indirect immunofluorescence test (IFT) etc [16].

Intradermal test (ID): In the Intradermal test presence of antibodies in patients against Schistosoma japonicum (or egg) antigen are detected. Antigen is diluted and administered to patients through intradermal injection. If a patient is positive there will be an intradermal reaction. This will indicate the patient likely has schistosomiasis [15]. This method is more reliable in early diagnosis.

Indirect hemagglutination assay (IHA): The principle of IHA is to attach Schistosoma japonicum soluble egg antigen for adsorption on human O-type erythrocyte surface, making it to be a sensitized erythrocytes. When sensitized erythrocytes are exposed to antibody in the patient's serum under suitable conditions, antigen adsorbed on erythrocyte surface reacts with specific antibody and then forms macroscopic erythrocyte agglutination. IHA has higher diagnostic performance with schistosomiasis [17]. A test kit for IHA for mature schistosome has been developed and an animal model control experiment was established by Chengsong-Sun $[18,19]$.

Enzyme-linked immunosorbent assay (ELISA): ELISA is the main immunological test method with high sensitivity and specificity to diagnose schistosomiasis to date. It involves adsorbing soluble antigens or antibodies onto a solid support such as polystyrene. Then through an antigen-antibody binding specificity, there is a qualitative or quantitative detection of the immune reaction [20].

Existing applications include enzyme-linked immunosorbent assay for detection of Serum Antibodies which can combine with the soluble cercaria antigen of Schistosoma japonicum (SCA-ELISA). Others include, soluble egg antigen-ELISA test (SEA-ELISA) and AWA-ELISA, whose results are valuable in early diagnosis of schistosomiasis [21]. Wang Jie reported that the purified recombinant protein $\mathrm{Sj}$ GAPDH was used to establish a conventional enzym-linked immunosorbent assay (Sj GAPDH ELISA) and ELISA with biotin-avidin signal amplification ( $\mathrm{Sj}$ GAPDH BA ELISA) for detecting IgG antibodies against Schistosoma japonicum GAPDH [22]. The test has been reported to be effective in detecting specific antibodies against Schistosoma japonicum GAPDH. The test is also useful in monitoring changes in antibody levels before and after treatment against schistosomiasis.

Dipstick dye immuno-assay (DDIA): DDIA is a newly developed method for the detection of schistosomiasis. It relies on colloidal dye-labeled protein combined with the corresponding antibodies to develop colour. The method is easy to perform and has high sensitivity. It is also suitable for large-scale screening. Existing research has reported that SCA with colloidal dye as the detection antigen has been used to establish the SCA-DD1A method for the diagnosis of schistosomiasis. Comparative studies have shown that both SCA-DDIA and SEA-DDIA tests are valuable in the diagnosis of schistosomiasis [23].

Circunoval precipitin test (COPT): COPT is a unique method of schistosomiasis detection. However, its main disadvantage is the long reaction time and the low sensitivity. It is the gold-standard for the diagnosis of schistosomiasis due to Schistosoma mansoni [24].

Dot immunogold filtration assay: Dot immunogold filtration assay uses a nitrocellulose membrane as the carrier, gold colloid as a marker for qualitative detection of antibodies in human serum against Schistosoma japonicum egg [15]. This 
method has high sensitivity with easy operation, and suitable for large-scale epidemiological investigation.

Indirect immunofluorescence test (IFT): In IFT fluorescein combines with anti-immunoglobulin antibody after preparing the antigen slice to form the secondary antibody that is labelled by fluorescein. The specific binding of antigen-antibody is used to observe the dynamic changes of antigen and antibody in infected tissue [23]. IFT has good sensitivity and specificity.

\section{Nucleic acid tests}

Nucleic acid test has better specificity and sensitivity for detection of schistosomiasis. Latest research has developed many improved methods such as PCR, Multiplex PCR, Realtime Quantitative PCR, PCR-ELISA, Loop-mediated Isothermal amplification method (LAMP) and Gene Chip Test.

Polymerase chain reaction (PCR): Conventional PCR is used to extract the DNA of schistosome in patient's stool, urine and blood as template. Then a direct probe is used to detect the nucleic acid fragments of pathogen, hence determining whether the pathogen infection is present or not. The target fragment include miRNA from schistosome such as sma-miR-277, smamiR-3479-3p and sma-bantam [25-27], and miRNA from parasitifer during infection such as miR-223, miR-122, miR-21 and miR-34a $[28,29]$. The miRNAs help to distinguish eggpositive or egg-negative individuals. The above types of miRNA can be used as biomarkers for the diagnosis of schistosomiasis [30]. This method is specific, sensitive and convenient, and ideal as a molecular diagnostic technique.

Multiplex polymerase chain reaction: Multiplex Polymerase Chain Reaction is involves adding two or more pairs of primers in the same reaction to amplify multiple nucleic acid fragments for pathogen simultaneously, and it's more efficient than conventional PCR [31].

Real-time quantitative polymerase chain reaction: Realtime Quantitative Polymerase Chain Reaction involves adding fluorophores into the PCR reaction system. This is followed by real-time monitoring of the entire PCR process with fluorescent signals, and analysis the total amount of unknown template by standard curve or other demand. This is a highly sensitive nucleic acid quantitative detection technology. The test is based on PCR technology and its advantages include accurate, quantitative, without the need for gel electrophoresis. It has a good prospect of application in disease surveillance [32]. Guan Wei confirmed the reliability of Real-time PCR [33], and reported the method to be valuable in assessing the condition of patients.

Polymerase chain reaction-enzyme-linked immunosorbent assay (PCR-ELISA): PCR-ELISA is through ELISA technique after PCR amplification to detection using enzymelabeled antibodies hybridization in solid-phase or liquid-phase. This method involves three parts: amplification product, hybridization of probe, enzyme colour of conventional ELISA. Its main advantages include high sensitivity like PCR, specificity like nucleic acid probe, objectivity like ELISA reader [34].
Loop-mediated isothermal amplification: Loop-mediated Isothermal Amplification is a novel method of thermostatic nucleic acid amplification. It doesn't need some processes like thermal denaturation of template, temperature cycling and electrophoresis. There is just single specific primer designed for multiple regions of the target gene, nucleic acid amplification by DNA polymerase under constant temperature conditions (60-65), and amplification takes 15 to $60 \mathrm{~min}$ [35]. Song xu-ri had designed the LAMP primer of specific gene fragments from Schistosoma japonicum and established the visualization of nucleic acid thermostatic amplification reaction system [36]. The negative or positive results can be judged according to the colour change of the reaction system. This method is simple operation, specificity, high sensitivity and has been applied to the detection of Snail that Schistosoma japonicum infections.

Gene chip test: Gene Chip Test uses a special glass or silicon chip as a carrier and contains thousand nucleic acid probes on it. It can provide a wealth of information about gene sequences by fluorescent or current detection when the nucleic acid fragments such as DNA, cDNA, RNA from sample react with the nucleic acid probe [37]. Its main advantages include easy convenience, high sensitivity and specificity.

\section{Medical imaging examinations}

Chronic schistosomiasis has atypical clinical symptoms and many complications. Medical imaging examination is helpful for diagnosis and judgment of patient's condition. Common means of inspection include B-scan ultrasonography, Computed Tomography and Magnetic Resonance Imaging CT and MRI

B-scan ultrasonography: Through the B-scan ultrasonography, the normal liver in patients with early infection can be evaluated. The liver volume shrinks after liver fibrosis or liver cirrhosis. The right lobe of the liver becomes more obvious. The liver capsule changes into a wavy or sawtooth form. The liver parenchyma changes like the pattern of a map or becomes flocculate. There are calcified plaque or spots, and portal vein and splenic vein widens. In late patients with hydrogaster, a liquid dark area exists in the abdominal cavity. Patients with liver cancer show low echo or isoechoic mass in the liver [37,38].

Computed tomography (CT): With the transfer, deposition and death of eggs could result in calcification in the tissue of damaged organs. Through the CT detection is the abnormal ratio of liver lobe to calcification can be assessed. The degree of severity is related to the amount of schistosoma in liver. The portal vein would become calcified because of the eggs which deposited in the portal vein system during the migration. CT images show arc-shaped, punctate or full vessel wall of highdensity shadow [38,39]. The CT imaging features of calcified colon wall show ribbon and strip - shaped of high-density shadow. Calcification is normally parallel to the intestinal wall. This symptom is as a result of the deposition of eggs in the colon mucosa and submucosa. This can be used as a strong basis for diagnosis of schistosomiasis. Advanced schistosome cirrhosis leads to multiple low density lesions in the liver 
parenchyma, especially in severe cases with liver cancer $[39,40]$.

Magnetic resonance imaging (MRI): MRI is usually used for the diagnosis of cerebral schistosomiasis, and is always combined with the CT report. CT and MRI imaging can reveal different degrees of edema area, especially the typical signs "finger-like" edema that extends to subcortical white. Lowdensity edema is common, enhanced scan reveals eosinophilic abscess and acute nodules were uniform enhancement like patchygrit-like and nodular in cerebral cortex [40,41]

\section{Conclusion}

Table 1. Diagnostic methods to allow for future improvements and development of diagnostic test for schistosomiasis.

\begin{tabular}{|c|c|c|c|}
\hline Methods & Specificity & Sensitivity & Application \\
\hline Blood routine examination & Low & High & Routine Examination \\
\hline Excrement Examination & High & Low & Gold-standard \\
\hline Microscopic Examination Of Urine Sediment & High & Low & Gold-standard \\
\hline Colonoscopy Biopsy & High & High & Gold-standard \\
\hline Intradermal Test & Medium & High & Only reliable in early diagnosis \\
\hline Indirect Hemagglutination Assay & High & High & Easy operate \\
\hline Enzyme-linked Immunosorbent Assay & High & High & Diagnosis and assessment of the disease \\
\hline Dipstick Dye Immuno-assay & Medium & High & Use for large-scale screening \\
\hline Circunoval Precipitin Test & High & Low & $\begin{array}{l}\text { the gold-standard for the diagnosis of } \\
\text { schistosomiasis due to Schistosoma mansoni }\end{array}$ \\
\hline Dot Immunogold Filtration Assay & High & High & Use for large-scale screening \\
\hline Indirect Immunofluorescence Test & High & High & \\
\hline Polymerase Chain Reaction & High & High & Common inspection methods \\
\hline Multiplex Polymerase Chain Reaction & High & High & More efficient than PCR \\
\hline Real-time Quantitative Polymerase Chain Reaction & High & High & Application in disease surveillance \\
\hline PCR-ELISA & High & High & \\
\hline Loop-mediated Isothermal Amplification & High & High & $\begin{array}{l}\text { Detection of Snail that Schistosoma japonicum } \\
\text { infections }\end{array}$ \\
\hline Gene Chip Tests & High & High & Still under study \\
\hline Medical Imaging examinations & Low & Medium & \\
\hline
\end{tabular}

Schistosomiasis in China has been effectively controlled; however, the new reports still indicate that it is still a major problem, endangering people's health and social development. Atypical schistosomiasis symptoms and low specificity of diagnosis methods still present a huge diagnostic challenge to clinicians. To circumvent the obstacle, clinicians have to make themselves familiar with all kinds of symptoms of schistosomiasis. Early and correct diagnosis would lead to early treatment of cases of schistosomiasis. It is also important to develop new diagnostic methods and techniques for more accurate detection of schistosome infection. There is need to identify advantages and disadvantages of existing diagnostic methods to allow for future improvements and development of diagnostic test for schistosomiasis shown in Table 1.

\section{References}

1. Gryseels B. J Infect Dis Clin North Am. 2012;26:383-97.

2. World Health Organization. Schistosomiasis: progress report 2001-2011, strategic plan. 2013;17.
3. Wang ZQ, Wang Y, Jia LJ, et al. Schistosoma haematobium infection in workers returning from Africa to China. J Travel Med. 2013;20:256-8.

4. Kuai X, Zhou C, Pang Z, et al. An analysis of 61 cases of intestinal schistosomiasis misdiagnosd as inflammatory bowel disease. Chin J of Prac Int Med. 2016;36:686-7.

5. Jing Y, Shao X. Pathogenesis and treatment of liver fibrosis in schistosomiasis. J Trop Dis And Parasitol. 2010;8:54-8.

6. Zhang H, Kong P, Li C, et al. CT Findings of Chronic Hepatic Schistosomiasis and Its Complications. J Kun Med Univ. 2016;37:62-6.

7. Huang Ya. One case of chronic schistosomiasis died of hepatopancreatic cancer without treatment for a long time. J Math Med. 2014;27:244.

8. Chen X, Wu Gl, Sun X, et al. Modern parasitology. BeiJing:People Military MedPress. 2002;662-71. 
9. Mo L, Cai X, Wang Q, et al. Misdiagnose Schistosomiasis Haematobia as Bladder Neoplasms: Two Cases Report and Literature Review. Chin Gen Pract. 2017;20:1005-8.

10. Medeiros TB, Domingues AL, Luna CF, et al. Correlation between platelet count and both liver fibrosis and spleen diameter in patients with schistosomiasis mansoni. Arq Gastroenterol. 2014;51:34-8.

11. Caldeira K, Teixeira CF, Silveira MB, et al. Comparison of the Ka-to-Katz and Hehnintex methods for the diagnosis of schistosomiasis in a low-intensity transmission focus in Bandeirantes; parana, southern Brazil. Mem Inst Oswaldo Cruz. 2012;107:690-2.

12. Xu Z ,He Z,Wang Y, et al. Application of Parasep feces centrifuge tube method in the diagnosis of Schistosomiasis. J Pub Health and Prev Med. 2015;26:76-7.

13. Wang Y, Wang J, Zhang J, et al. Retrospective analysis of 2 cases of imported Schistosomiasis haematobia in Henan province. Chin Front Health Quarantine. 2017;40:188-90.

14. Nausch N, Dawson EM, Midzi N, et al. Field evaluation of a new antibody-based diagnostic for Schistosoma haematobium and $\mathrm{S}$. mansoni at the point of care in northeast Zimbabwe. BMC Infect Dis. 2014;14:165.

15. Wng X, Cai A, Zhou M. Research Status of Experimental Diagnosis of Schistosomiasis. Int $\mathrm{J}$ Lab Med. 2016;37:1824-6.

16. Wu Z, Liang L, Jiang Q, et al. Case of intracranial schistosomiasis granuloma was misdiagnosed as brain metastases. Chin J Clin Neurosurg. 2017;22:286.

17. Wang L, Wang Y, Wei D. Efficiency of indirect hemagglutination assay (IHA) for the diagnosis of Schistosoma japonicum human infections: a metaanalysis. J Pathog Biol. 2017;12:155-9.

18. Sun C, Wang F, Wang Y, et al. Preliminary evaluation of an indirect hemagglutination assay kit on Site screening of schistosomiasis japonica. J Pathog Biol. 2013;8:982-5.

19. Sun C, Zhang L, Zhu H, et al. Preliminary evaluation of an indirect hemagglutination assay kit on early diagnosis of schistosomiasis japonica. J Trop Dis and Parasitol. 2016; $14: 125-7$.

20. Othman RA. Indirect haemagglutination test and ELISA as compared to Kato thick-smear in diagnosing Schistosoma mansoni. J Egypt Soc Parasitol. 2013;43:841-8.

21. Si W, Wang T. Progress of researches on diagnostic antigens of schistosomiasis japonica. Chin J Schisto Control. 2012;24:345-9.

22. Wang Jie, Liu Xi, Zhang Q, et al. The value of diagnosing schistosomiasis by detecting $\operatorname{IgG}$ antibodies against glyceraldehyde-3-phosphate dehydrogenase of Schistosoma japonicum. J Pathog Biol. 2015;10:976-9.

23. Jin $\mathrm{J}, \mathrm{Xu} \mathrm{j}, \mathrm{Xu} \mathrm{S}$, et al. Advances in the study of early diagnosis of schistosomiasis. $\mathrm{J}$ Pathog Biol. 2013;8:1142-7.

24. Noya O, Alarcon D, Losada S, et al. Laboratory diagnosis of Schistosomiasis in areas of low transmission; a review of a line of research. Mem lnst Oswaldo Cruz. 2002;97:167-9.

25. Burke ML, Mcmanus DP, Ramm GA, et al. Temporal expression of chemokines dictates the hepatic inflammatory infiltrate in a murine model of schistosomiasis. PLoS Negl Trop Dis. 2010;4:598.

26. ChenX, Lung 1l, Ghang J, et al. Seoreted mioroRNAs: a new form of intercellular communication. Trends Cell Biol. 2012;22:125-32.

27. Cheng $\mathrm{G}$, Luo $\mathrm{R}, \mathrm{Hu} \mathrm{C}$, et al. Deep sequencing-based identification of pathogen-pecific microRNAs in the plasma of rabbits infected with Schistosoma japonicum. 2013;140:1751-61.

28. He X, Sai X, Chen C, et al. Host serum miR-223 is a potential new biomarker for Schistosoma japonicum infection and the response to chemotherapy. Parasit Vectors. 2013;6:272.

29. Cai P, Gobert GN, Hong Y, et al. Circulating miRNAs: Potential Novel Biomarkers for Hepatopathology progression and Diagnosis of Schistosomiasis Japonica in Two Murine Models. PLoS Negl Trop Dis. 2015;9:e0003965.

30. Hoy AM, Lundie RJ, Ivens A, et al. Parasite-derived microRNAs in host serum as novel biomarkers of helminth infection. PLoS Negl Trop Dis. 2014;8:e2701.

31. Caliendo AM. Multiplex PCR and emerging technologies for the detection of respiratory pathogens. Clin lnfect Dis. 2011;2:326-30.

32. O'connor L, Glynn B. Recent advances in the development of nucleic acid diagnostics. Expert Rev Med Device. 2010;7:529-39.

33. Guan W, Xia C. Real-time PCR for dynamic changes of the serum DNA in hosts infected with $\mathrm{S}$. japonicum and diagnosis and therapy evaluation of schistosomiasis. Suzhou University. 2013.

34. Comes LI, Dos Santos MI, Enk MJ, et al. Development and evaluation of a sensitive PCR-ELISA system for detection of Schistosoma infection in feces. PLoS Negl Trop Dis. 2010;4:664.

35. Thekisoe OM, Kuboki N, Nambota A, et al. Species-specific loop-mediated isothermal amplification (LAMP) for diagnosis of trypanosomosis. Acta Trop. 2007;102:182-9.

36. Lu Y, Shi H, Li C. Current status and research progress of clinical examination on schistosomiasis. Int J Med Parasit Dis. 2015;42:236-9.

37. Sun B. Diagnostic value of liver ultrasound in schistosomiasis. Health way. 2016;15:252-3.

38. Wu Z, Liang L, Jiang Q, et al. 1 case of intracranial schistosomiasis granuloma was misdiagnosed as brain metastases. Chin J Clin Neurosurg. 2017;22:286.

39. Yuan K, Li W. Correlative Study on Clinic and CT Features of Advanced Schistosomiasis Cirrhosis. Chin J Ctrl Endem Dis. 2016;31:1300.

40. Zhu F, Huang X, Wu M, et al. Diagnosis and treatment of cerebral schistosomiasis: a report of 166 cases. Chin J Schisto Control. 2014;26:695-6. 
Citation: Yumin Z, Weifeng G, Joseph WM et al.. Advances in diagnosis of schistosomiasis. Microbiol Curr Res. 2018;2(2):3-8.

\section{Correspondence to:}

Yumin Zhao

Department of Parasitology

Guilin Medical University,

Guilin, Guangxi, China

E-mail: zymcsg@sina.com

\section{Zhisheng Dang}

Key Laboratory on Biology of Parasite and Vector, Ministry of Health,

Shanghai, China

E-mail: dnagzs@nipd.chinacdc.cn 